Industrial Health, 1984, 22, 189-198

\title{
Electrocorticogram (ECoG) and Auditory Evoked Potential (AEP) in Rats Intoxicated with Lead Acetate
}

\author{
Yasuo TAKAHASHI, ${ }^{1 *}$ Yasunori OKAMOTO $^{1}$ and Kazuo SAITO ${ }^{2}$ \\ ${ }^{1}$ Department of Environmental Medicine, Graduate School of Environmental Science and \\ ${ }^{2}$ Department of Hygiene and Preventive Medicine, Hokkaido University \\ School of Medicine, Kita-15, Nishi-7, Kita-ku, Sapporo, 060 Japan
}

(Received March 12, 1984 and in revised form June 7, 1984)

\begin{abstract}
ECoG and AEP of male Sprague-Dawley rats were recorded to elucidate the effect of lead acetate on the central nervous system. Lead acetate $\left(1.0 \times 10^{-2} \mathrm{M}\right)$ in distilled water, $1 \mathrm{ml} / 100 \mathrm{~g}$ body weight, was administered intraperitoneally for four consecutive days to an experimental group of rats. On the 11th day after injection, the rats were decapitated and the content of lead in the whole brain was measured in addition to the blood lead concentration and the hematocrit. The body weights and hematocrit values of rats in the experimental group decreased remarkably although they tended to recover by the end of the experimental period. The blood lead concentration of rats in the experimental group increased throughout the experimental period and their whole brain lead amount was twelve times that of the control group. No changes in the ECoG of rats given lead acetate were found in this experiment, but the changes in AEP of the rats in the experimental group were delayed P2 peak latency and increased P1-N1 peak to peak amplitude.
\end{abstract}

Key words: Lead intoxication-AEP-ECoG

\section{INTRODUCTON}

There have been a number of reports about the effects of lead poisoning. Anemia, fatigue, headache, and myalgia are seen as the effects of lead in the early stages, while at more advanced stages, lead poisoning causes brain malfunction. The cerebellum in particular is easily damaged. ${ }^{1-5)}$ These effects can be seen in the child more often than in the adult. ${ }^{3,6,7)}$ Among biochemical studies, many effects of lead have been reported; for example, there is a decrease in ALA-D activity ${ }^{7,8)}$ and an increase in noradrenaline in the whole brain of rats. ${ }^{9)}$ Furthermore, in young rats there is an increase in noradrenaline and serotonin in the midbrain, and increases in dopamine in the midbrain and striatum following

* Present address: Tokyo Metropolitan Institute of Gerontology, 35-2 Sakae-cho, Itabashiku, Tokyo 173, Japan.

Reprint requests to: Prof. K. Saito. 
the injection of lead acetate have been reported. ${ }^{10)}$ In children, low voltage ${ }^{11)}$ and changes in EEG spectra ${ }^{12)}$ have been observed after exposure to lead. In animals, rats injected with leaded petrol have shown an increase of $\theta$ and $\alpha$ waves on $\mathrm{ECoG},{ }^{13)}$ while rats administered leaded gasoline have indicated a characteristic delay in latency and a decreased amplitude in auditory evoked potentials. ${ }^{14)}$ It is also clear that lead inhibits synaptic transmission. ${ }^{15}$ ) Nevertheless, current studies of the effects of lead on EEG and AEP are still inadequate. In this study, therefore, the effect of lead acetate on the central nervous system of rats was analyzed in order to help clarify these effects more fully.

\section{Materials ANd Methods}

The skulls of 10 male Sprague-Dawley rats, weighing 230 to $320 \mathrm{~g}$ and aged 11 weeks, were drilled at points about $2 \mathrm{~mm}$ from the right side of the sagittal suture and $2 \mathrm{~mm}$ above and below the coronary suture under Nembutal anesthesia. Bipolar electrodes, having a small socket with a silver globular tip $0.7 \mathrm{~mm}$ in diameter to protect the brain cortex from injury, were implanted on the surface about $2 \mathrm{~mm}$ apart over the frontal and occipital lobes of the right hemisphere of the brain. The electrodes were attached permanently on the skull with dental cement. The observations were made one month after recovery from the implant operation. The rats were divided into two groups, a control group containing four rats and an experimental group of six rats. The control group was given distilled water only, whereas the experimental group was given $1.0 \times 10^{-2} \mathrm{M}$ lead acetate in distilled water intraperitoneally in a dose of $1 \mathrm{ml} / 100 \mathrm{~g}$ body weight. In both groups, the injections were performed for four consecutive days. ECoG and AEP were recorded on the day before lead administration, and on the 1st, 2nd, 3rd, 6th, 9th and 11th days after the final administration under normal conditions without restriction in an electrically shielded chamber. The ECoG was recorded and a fast fourier transformation (FFT) of these waves was performed at 4-second intervals; the mean average over 1 minute was taken and the power spectrums were obtained.

From these power spectrums, we obtained the power of each $\delta$ wave: $2-4 \mathrm{~Hz}$; $\theta$ wave: $4-8 \mathrm{~Hz} ; \alpha$ wave: $8-13 \mathrm{~Hz} ; \beta$ wave: $13-20 \mathrm{~Hz}$; as well as the predominant wave: $6-9 \mathrm{~Hz}$, and the percent values of each wave to the total power.

Averaged auditory evoked potentials (AEP) to a series of 100 sound clicks presented at a repetition of 0.5 times per second, $78 \mathrm{~dB}$ hearing level, $1000 \mathrm{~Hz}$ in frequency and $50 \mathrm{msec}$ in duration were computed using a microcomputer (Nihon Koden Model ATAC-450). The clicks were produced by an audio signal generator (JEIC Type $01001 \mathrm{~A}$ ) connected to an electronic stimulator (Nihon Kohden Model SEN-7103). The peak latencies and the peak-to-peak amplitudes of AEP were measured.

After several measurements each of ECoG and AEP had been taken, blood 
was removed from the tail vein of the rats, and the hematocrit values and the blood lead concentrations were measured. At the end of the experimental period, the rats were decapitated, and their brains were removed. The blood lead concentrations and lead content in the whole brain of the rats were determined by atomic absorption spectrophotometry (Hitachi Model 170-70).

\section{RESUlTS}

The changes in body weight of the rats in both the control and experimental groups are shown in Fig. 1. The body weights of the experimental group obviously decreased after injection. The body weights gradually recovered from the 7 th day after injection, but at the end of the experimental period they had not regained the levels of the control group. The changes in blood lead concentration are shown in Fig. 2. Blood lead concentrations in the rats of the control group were less than $10 \mu \mathrm{g} / \mathrm{d} l$ throughout the experimental period, whereas those of the experimental group were $267 \pm 19 \mu \mathrm{g} / \mathrm{d} l$ (mean \pm S.E.) on the 1 st day after injection. These increased gradually until the end of the experimental period, when they reached a concentration of $697 \pm 139 \mu \mathrm{g} / \mathrm{d} l$ (mean \pm S.E.).

Fig. 3 indicates the changes in hematocrit. The values in the experimental group decreased gradually, and on the 9th day after injection they reached their lowest level, $33.0 \pm 1.1 \%$ (mean \pm S.E.); at the end of the experimental period, they showed recovery. As shown in Fig. 4, the lead content in the brains of the experimental group was $2.076 \pm 0.133 \mu \mathrm{g} / \mathrm{g}$ wet weight (mean \pm S.E.), a value

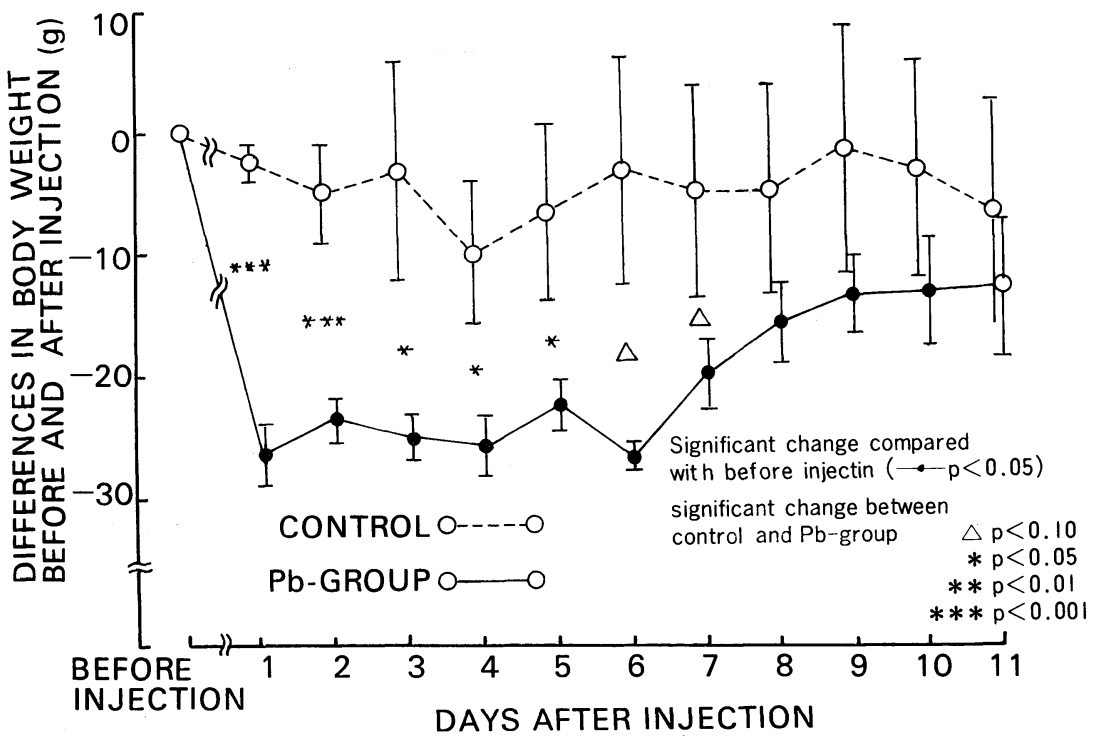

Fig. 1. Changes in body weight in control and Pb-group (mean \pm S.E.) 


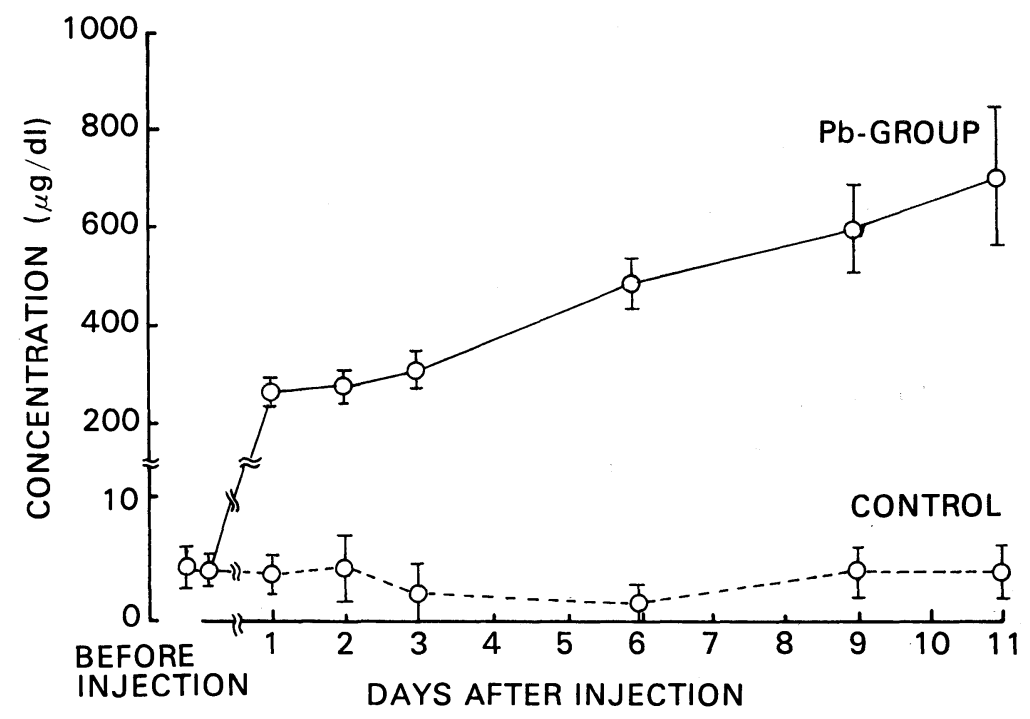

Fig. 2. Changes in blood lead concentration in control and Pb-group $($ mean \pm S.E. $)$.

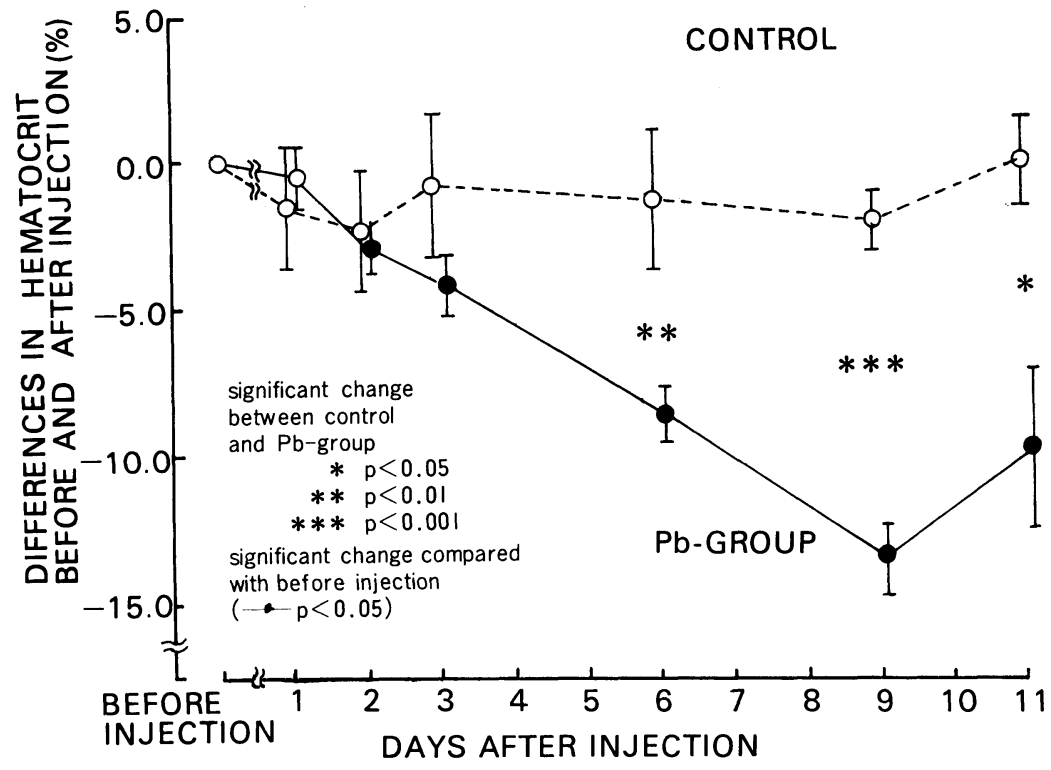

Fig. 3. Changes in hematocrit in control and $\mathbf{P b}$-group (mean \pm S.E.).

twelve times greater than that of the control group $[0.175 \pm 0.025 \mu \mathrm{g} / \mathrm{g}$ wet weight (mean \pm S.E.)]. Upon dissection of the rats at the end of the experimental period, no abnormalities were recognized in the control group. In the experimental group, however, adhesion of the abdominal organs was observed and these organs 


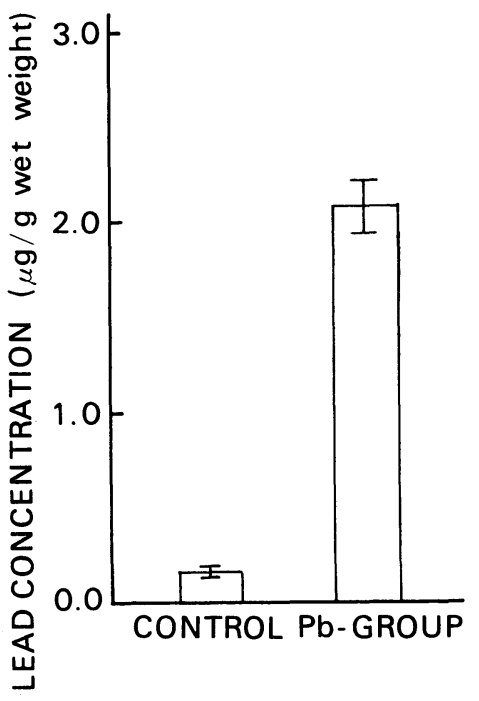

Fig. 4. Brain lead concentration of rats 11 days after injection (mean \pm S.E.).

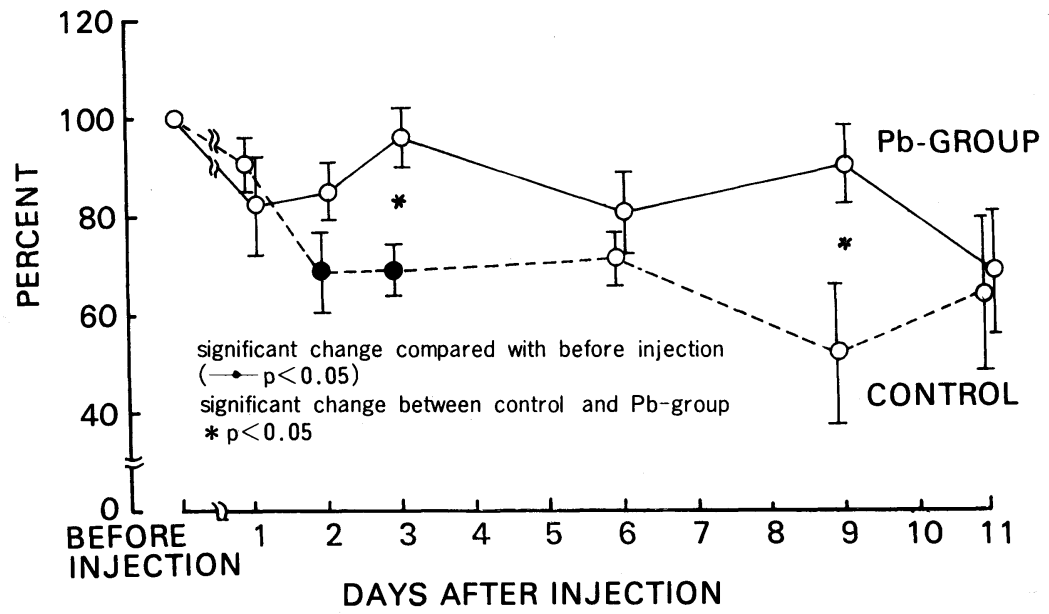

Fig. 5. Changes in brain wave power $(6-9 \mathrm{~Hz})$ in control and $\mathbf{P b}$ group (mean \pm S.E.)

had been covered by white membranous matter. The results of ECoG analysis showed that the $\theta(4-8 \mathrm{~Hz})$ wave component of ECoG in all rats was more than $50 \%$. In the four wave components, $\delta, \theta, \alpha$ and $\beta$, the experimental group showed no significant differences. For all rats, the $6-9 \mathrm{~Hz}$ component was predominant, and Fig. 5 shows the changes in $6-9 \mathrm{~Hz}$ power in both the control 


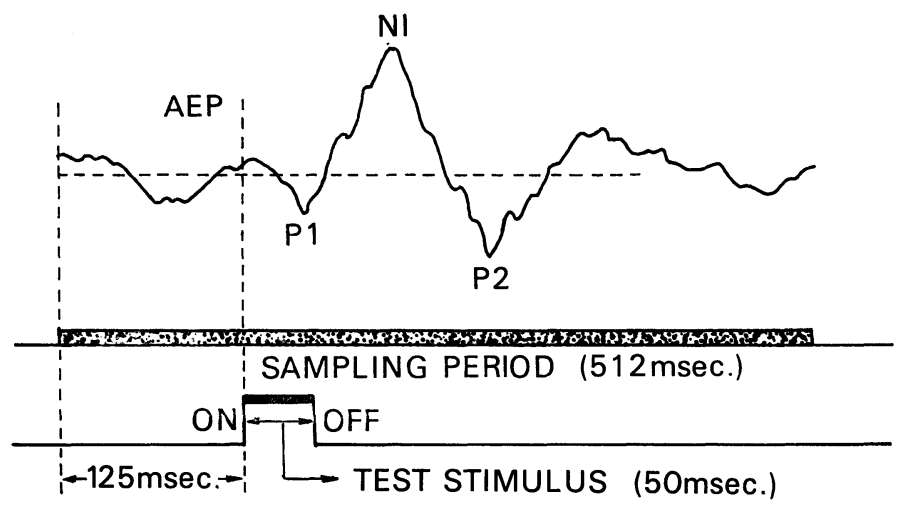

Fig. 6 Sampling period and identification of AEP components.

and experimental groups. On the 3rd and 9th days after injection, significant differences between the control and experimental groups were obtained. In both groups, $6-9 \mathrm{~Hz}$ power was apt to decrease after injections. Fig. 6 shows the typical AEP of rats before lead acetate injection and the analytical procedure. The mean peak latencies of the first positive wave (P1), the first negative wave (N1) and the second positive wave (P2) were 35.3, 97.8 and $166.9 \mathrm{msec}$, respectively. The peak-to-peak amplitudes of P1-N1 and N1-P2 were $13.5 \mu \mathrm{V}$ and $13.5 \mu \mathrm{V}$ on average. Throughout the experimental period, there were no significant differences between the control and the experimental groups in P1 latncy. On the 2nd day after injection, the changes in $\mathrm{N} 1$ latency in the experimental group increased significantly $(p<0.05)$ compared with the latency before injection. There tended to be a significant difference between the control and experimental groups in the changes of $\mathrm{N} 1$ latency on the 3rd and 9th day after injection. Fig. 7 shows that the changes in P2 latency of the experimental group increased significantly $(\mathrm{p}<0.05)$ on the 9 th day after injection in comparison with those prior to injection; there was also a tendency for a significant difference to exist between the control and experimental groups on the 3rd, 6th and 11th days after injection.

As shown in Fig. 8, the P1-N1 amplitude in the experimental group increased significantly $(p<0.05)$ in comparison with that before injection on the 1 st and 6th days after injection; there was also a tendency for a significant difference to exist between the control and experimental groups on the 1st, 9th and 11th days after injection as well as on the 6th day after injection. The changes in N1-P2 amplitude in the control group were significantly increased on the 1st day after injection when compared with those prior to injection; there was no significant difference between the control and experimental groups throughout the experimental period. 


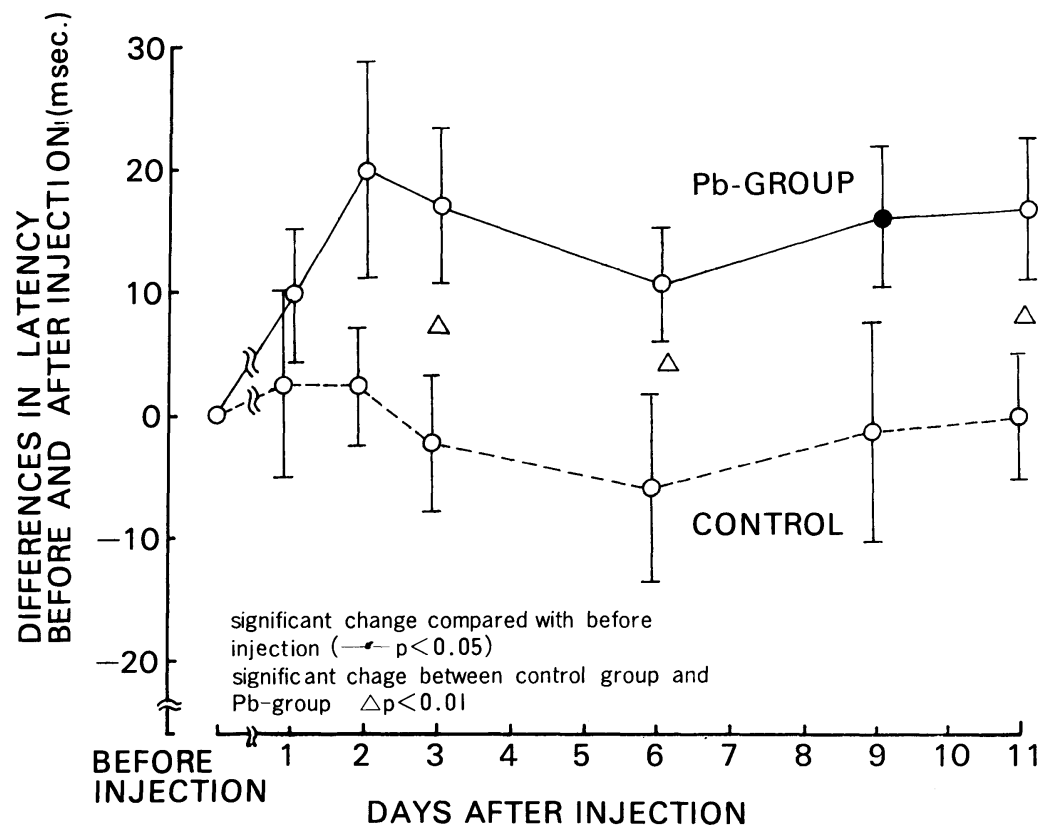

Fig. 7. Changes in P2 latency in control and Pb-group (mean \pm S.E.).

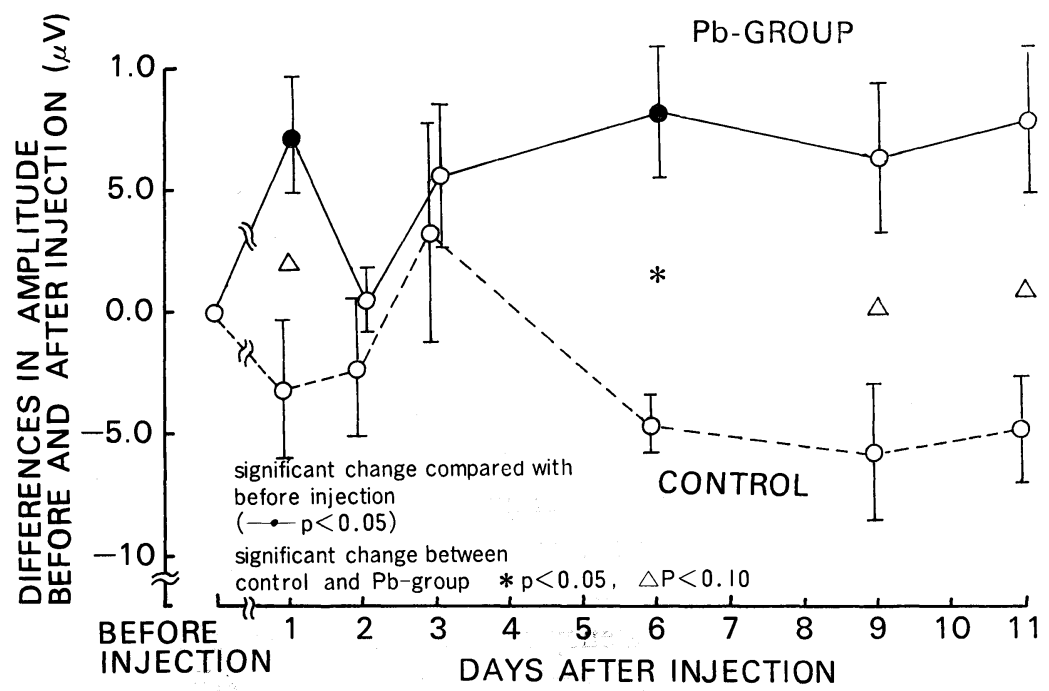

Fig. 8. Changes in P1-N1 amplitude in control and Pb-group (mean \pm S.E.). 


\section{Discussion}

In this study, lead acetate $\left(1.0 \times 10^{-2} \mathrm{M}\right)$ was administered intraperitoneally for four consecutive days to rats in an experimental group, $1 \mathrm{ml} / 100 \mathrm{~g}$ body weight. The total dose of injection was $152 \mathrm{mg} / \mathrm{kg}$ body weight, greater than the dose of $\mathrm{LD}_{50}(130 \mathrm{mg} / \mathrm{kg}$ body weight $)$. Changes in blood lead concentration of the rats in the experimental group increased throughout the experimental period, but changes in body weight and hematocrit decreased after injection, although they showed a tendency to recover by the end of the experimental period. The whole brain lead concentration in the lead group was $2.076 \pm 0.133 \mu \mathrm{g} / \mathrm{g}$ wet weight (mean \pm S.E.) on the 11 th day after injection. In previous studies, the brain lead uptake has been proportional to the dose after intravenous injection and the brain lead retention has been prolonged, although blood lead levels have fallen rapidly after injection. ${ }^{3)}$ We inferred from these results that the brain lead volume is likely to increase further under our experimental conditons after the experimental period.

The purpose of this study was to investigate the effect of lead acetate on the central nervous system of rats by measuring both ECoG and AEP. Analysis of ECoG power showed that the $6-9 \mathrm{~Hz}$ wave component was predominant among free rats. The changes in the potential power of the $6-9 \mathrm{~Hz}$ component in both groups showed a tendency to decrease when compared with those prior to injeciton. From this result, we assumed that the effect of the injections was more clearly apparent than the effect of lead acetate on the ECoG of these rats. Saito (1973) ${ }^{13)}$ reported that after the injection of nonleaded white petrol (WP) and leaded petrol (LP) to rats, the $\theta(4-8 \mathrm{~Hz})$ and $\alpha(8-13 \mathrm{~Hz})$ wave power decreased until the 3rd day after injection, whereas the $\theta$ and $\alpha$ wave power of the LP group increased rapidly on the 10 th day after injection. We deduced from this result that the total lead acetate volume of injections in our experiment was lower and the experimental period was shorter in order to promote significant changes in rat ECoG.

In the analysis of AEP latencies, a delay in P2 latency was clearly apparent in the experimental group. There were no significant differences in changes in P1 latency. We assumed from these results that AEP response time was delayed by the injection of lead acetate. Although there were significant differences in the N1-P2 amplitudes of both groups in comparison with the pre-injection condition on only the 1st day after injection, the changes in P1-N1 amplitudes in the experimental group increased on the 1st, 6th, 9th and 11th days after injection. It was interesting to note that P2 latency as a late component of AEP was affected, while the P1-N1 amplitude as an earlier component of AEP was influenced. Previous reports have indicated that the peripheral nerve conduction velocity of humans and mammals is lowered by the injection of lead compounds. ${ }^{16,17)}$

Other published studies have noted that the strength of muscle contraction is 
also lowered and that the latency of the contraction is delayed at the nerve-muscle junctions by injection of a lead compound. ${ }^{18)}$ Further experiments have reported that the presynapitc regions of neurons are more affected by the injection of a lead compound than the postsynaptic regions and that lead inhibits the release of neurotransmitters. ${ }^{15)}$ The delay in AEP latency therefore would be estimated from the synaptic inhibition of the lead compound. However, it was difficult to explain why the delay affected only the P2 peak component while the P1-N1 amplitude was increased.

Many previous reports have indicated morphological abnormalities of the brain following the injection of a lead compound. In particular, the offspring of mammals have often been studied, and it is well known that the young mammal is more seriously damaged by the injection of a lead compound than the adult. ${ }^{3-8}$ ) In this study, however, we used 15-week-old rats, an age at which the growth of their brains was considered to be complete, and no morphological brain abnormalities could be observed. In lead poisoning in children, brain hemorrhage and edema have been clearly observed, ${ }^{3-4,6)}$ and the cerebellum has been most severely damaged. ${ }^{3-5)}$

Other reports have indicated that morphological abnormalities of the cerebellum capillary vessels can be observed, that its membrane permeability is affected, ${ }^{3)}$ and that, in addition, hypervascularization is produced in the cerebral cortex of rats ${ }^{19)}$ by the injection of a lead compound. Because the cerebellum plays an important role in the production of movement and the regulation of posture, ataxy and a permanent bending posture may be important indices of lead poisoning. Further, we have to consider the transmission of lead from the blood to the brain. Some reports have indicated that the blood-brain barrier (BBB) of the child is damaged by the injection of a lead compound. ${ }^{20,21)}$ We considered from these results that the transmission of lead from the blood to the brain tissue in a child is so easy that, consequently, the lead volume in the brain will be increased and that striking brain damage will appear. As the development of BBB of an unborn baby is incomplete, being finished only after birth, we can easily understand why the child is more affected than the adult by the injection of a lead compound. Our experimental period was 15 days, and we must take the length of this observational period into consideration, because the rats in this experiment were adult and the blood-lead concentration was still increasing by the end of the experimental period. We therefore felt the need of a longer experimental period. It is also important to consider the relation between the threshold of the physiological phenomena by the injection of a lead compound and the lead level of both blood and brain. 


\section{REFERENCES}

1) Pentschew, A. (1965). Morphology and morphogenesis of lead encephalopathy, Acta Neuropathol., 5, 133.

2) Pentschew, A. and Garro, F. (1966). Lead encephalomyelopathy of the suckling rat and its implications on the porphyrinopathic nervous diseases. With special reference to the permeability disorders of the nervous system's capillaries, Acta Neuropathol., 6, 266.

3) Goldstein, W., Asbury, K. and Diamond, I. (1974). Pathogenesis of lead encephalopathy, Arch. Neurol., 1, 382.

4) Toews, D., Kolber, A., Hayward, J., Kringman, R. and Morell, P. (1978). Experimental lead encephalopathy in the suckling rat: concentration of lead in cellular fractions enriched in brain capillaries, Brain Res., 147, 131.

5) Michaelson, A. and Sauerhoff, W. (1974). An improved model of lead-induced brain dysfunction in the suckling rat, Toxicol. Appl. Pharmacol., 28, 88.

6) Krigman, R., Mushak, P. and Bouldin, W. (1977). An appraisal of rodent models of lead encephalopathy, Neurotoxicology, Raven Press, New York, 1, 299.

7) Barlow, J., Baruah, K. and Davison, N. (1977). $\delta$-Aminolaevulinic acid dehydratase activity and focal brain haemorrhageas in lead-treated rats, Acta Neuropathol., 39, 219.

8) Millar, A., Battistini, V., Cumming, C., Carswell, F. and Godberg, A. (1970). Lead and $\delta$-aminolaevulinic acid dehydratase levels in mentally retarded children and in lead-poisoned suckling rats, Lancet, 3, 695.

9) Stija, K. and Seth, D. (1978). Dopamine and noradrenaline levels in the brains of lead and zinc poisoned rats, Toxicology, 10, 13.

10) Dubas, C., Stevenson, A., Singhal, L. and Hrdina, D. (1978). Regional alterations of brain biogenic amines in young rats following chronic lead exposure, Toxicology, 9, 185.

11) Burrows, E., Redle-Short, J. and Hanna, D. (1951). Lead poisoning in children, Brit. Med. J., 1, 329.

12) Benignus, V. A., Otto, D. A., Muller, K. E. and Seiple, K. J. (1981). Effects of age and body lead burden on CNS functoin in young children. II EEG spectra, Electroencephalog. Clin. Neurophysiol., 52, 240.

13) Saito, K. (1973). Electroencephalographic studies on petrol intoxication: comparison between nonleaded and leaded white petrol, Br. J. Ind. Med., 30, 352.

14) Saito, K., Kamita, N. and Takakuwa, E. (1974). Averaged auditory evoked response (AER) in rat intoxicated with gasoline, Ind. Health, 12, 97.

15) Manalis, S. and Cooper, P. (1973). Presynaptic and postsynaptic effects of lead at the frog neuromuscular junction, Nature, 243, 354.

16) Fullerton, M. (1966). Chronic peripheral neuropathy produced by lead poisoning in guinea pigs, J. Neuropathol. Exp. Neurol., 25, 214.

17) Takeuchi, H., Takahashi, M., Hara, I., Hashimoto, K., Suenaka, T. and Genba, H. (1975). An electrophysiological study on peripheral nerve injuries of workers handline lead, Jpn. J. Ind. Health, 17, 467.

18) Silbergeld, K., Fales, T. and Goldberg, M. (1974). The effects of inorganic lead on the neuromuscular junciton, Neuropharmacology, 13, 795.

19) Reyners, H., Reyners, G. and Maisin, R. (1976). Hypervascularization of the cerebral cortex in lead-induced encephalopathy, Experientia, 32, 1416.

20) Cremer, J. E., Braun, L. D. and Oldendorf, W. H. (1976). Changes during development in transport processes of the blood-brain barrier. Biochem. Biophys. Acta, 448, 633.

21) Feldman, G., Hayes, K., Youne, R. and Aldrich, D. (1977). Lead neuropathy in adults and children, Arch. Neurol., 34, 481. 